\title{
Absolute Quantification of Fatty Acid and Proximate Composition of Cow and Goat Powdered Milks
}

\author{
Weliton P. Batiston, Swami A. Maruyama, Sandra T. M. Gomes, Jesuí V. Visentainer, \\ Nilson E. de Souza and Makoto Matsushita*
}

Departamento de Química, Universidade Estadual de Maringá, Av. Colombo, 5790, 87020-900 Maringá-PR, Brazil

\begin{abstract}
Este trabalho investigou a qualidade lipídica de leites processados por quantificação de ácidos graxos. Dez amostras de leite em pó integral de vaca e duas de cabra foram submetidas à extração de lipídios totais e à preparação dos ésteres metílicos de ácidos graxos. Estes foram separados em cromatógrafo a gás. A variação da quantidade de ácidos graxos por gramas de lipídios para compostos poliinsaturados, ômega 6 , ômega 3 e ácido linoléico conjugado, foi respectivamente: 25,20-46,67, 12,82-31,45, 1,77-7,65 e 7,77-11,60 mg g-1 . A razão n-6/n-3 foi menor para os leites em pó de vaca $(2,85-6,07)$ em relação aos de cabra $(11,31-12,75)$ e a quantidade de ácidos graxos trans variou de 0,09 a $0,17 \mathrm{~g}$ por porção de $26 \mathrm{~g}$. As amostras apresentaram ácidos graxos essenciais ômega 6 e ômega 3, isômeros do ácido linoléico conjugado e quantidade de ácidos graxos trans em conformidade com a legislação brasileira.
\end{abstract}

This work investigated of the lipid quality of processed milks by fatty acid quantification. Ten samples of dry whole milk from cow and two from goat were submitted to total lipid extraction and to fatty acid methyl ester synthesis. Then, the synthesized products were separated in a gas chromatograph. The variation of the fatty acid per total lipid gram for polyunsaturated compounds, omega-6, omega-3 and conjugated linoleic acid, was 25.20-46.67, 12.82-31.45, 1.77-7.65 and 7.77-11.60, respectively. The $\mathrm{n}-6 / \mathrm{n}-3$ ratio for the cow powdered milk samples (2.85-6.07) was lesser reagarding goat milk samples (11.31-12.75) and the trans fatty acid amount varied from 0.09 to $0.17 \mathrm{~g}$ per $26 \mathrm{~g}$ portion. Essential fatty acids from the omega- 3 and omega- 6 groups were present in every sample, besides conjugated linoleic acid isomers. The trans fatty acid contents of the samples is in accordance with the Brazilian legislation.

Keywords: processed milk, omega-3, CLA, gas chromatography

\section{Introduction}

Milk is necessary in the diet of all the human beings, regardless of their age, and it has an important role in the proper growth of children due to its high nutritional value composed of lipids (essential fatty acids), carbohydrates (lactose), proteins (casein), vitamins ( $\mathrm{A}, \mathrm{B}_{1}, \mathrm{~B}_{2}, \mathrm{~B}_{6}, \mathrm{~B}_{12}$, $\mathrm{C}, \mathrm{D}, \mathrm{E}$ and $\mathrm{K})$ and mineral substances $(\mathrm{Ca}, \mathrm{P}, \mathrm{Mg}, \mathrm{Fe}$, $\mathrm{Cu}$ and $\mathrm{Zn}) .^{1,2}$

The lipids in the milk of ruminants are a great conjugated linoleic acid (CLA) source. It is a trans fatty acid (TFA) that represents a mixture of positional and geometric isomers of linoleic acid. ${ }^{3}$

\footnotetext{
*e-mail:mmakoto@uem.com.br
}

Many researches indicate that there is an antioxidant molecular mechanism, preventing tumor DNA generation, suppressing carcinogenesis activation, producting immunostimulatory effects and possessing antithrombotic, anti-obesity and anti-diabetic properties. ${ }^{4}$

Milk contains essential fatty acids (FA) from the omega-6 (n-6) and omega-3 (n-3) group, which are respectively: the linoleic acid (LA) and the alpha-linolenic acid (LNA). Both are necessary to keep cell membranes, cerebral functions and nerve impulse transmissions under normal conditions. These FA also participate in the atmospheric oxygen transference to the sanguine plasma, hemoglobin synthesis and cell division. ${ }^{5}$

Especially, LNA is converted into eicosapentaenoic acid (EPA) through the chain desaturation and elongation 
processes that allow eicosanoids production. These compounds that are essential to inflammatory and immunologic response enhancement and reduction of lipids in blood. ${ }^{6}$ Besides that, they possess vasodilator properties and might inhibit cancer development. ${ }^{7}$

The advances in the techniques directed to the steps of milk production, processing and distribution enhance the human acceptability of the final product, especially the milk of bovine origin. However, these steps might change its physical properties. ${ }^{8,9}$

Among the products of different industrial processes aimed to the achievement of suitable milks for commercial purposes, the powdered milk is very important due to its great shelf life (one year, approximately). This longevity is justified by its low moisture content, which inhibits the microorganism growth, removing the refrigeration need for its conservation. ${ }^{10}$

To obtain powdered milk, first the in natura milk is submitted to temperatures between 72 and $75^{\circ} \mathrm{C}$ for a period of time that might vary from 15 to $20 \mathrm{~s}$, and cooled between 5 and $7{ }^{\circ} \mathrm{C}$ right after. This process is called pasteurization. Then it suffers evaporation, through its runoff in vertical metallic walls heated to $77^{\circ} \mathrm{C}$. The concentrated product is atomized in a spray dryer, which sprinkles an air current at a temperature of $180^{\circ} \mathrm{C} \cdot .^{10,11}$

It is proved that foods submitted to high temperatures in the presence of oxygen may suffer oxidative reactions, with the lipid degradation showing a great importance among them. ${ }^{12}$ Fatty acids with a high number of unsaturated carbons will oxidize with a greater intensity than the ones with a lesser degree of unsaturations. ${ }^{9}$

This happens due to the fact that the oxygen, either from the atmosphere or from the regions around a dissolved sample, reacts with the most reactive segments of a triacylglycerol molecule, the doubly-bonded carbons of the FA carbonic chain and free radicals are the products of this reaction. This kind of compound can also be obtained through radiation absorption, thermal treatment and reaction with metallic ions or with other free radicals. ${ }^{9}$

Other researches indicate that food emulsions (oil-water interface) possess great impact over the lipid oxidation mechanisms due to its large superficial area, which enhances the lipid interaction with oxidizing substances that are soluble in water. ${ }^{13}$

This present work aimed for the profile evaluation and absolute quantification of the main FA, especially the polyunsaturated fatty acids (PUFA) that are found in milk lipids, and are the essential LA (18:2n-6), (18:3n-6) and LNA (18:3n-3), in addition to CLA isomers and trans FA. Proximate composition (moisture, ash, protein, total lipids and carbohydrates) was also determined. Another objective of this work was the characterization of lipid and physicochemical properties that may vary between the cow and goat powdered milks.

\section{Experimental}

\section{Sampling}

Whole powdered milks from twelve different brands were purchased from the local market, in Maringá City, Paraná State, Brazil, ten of bovine origin (denominated V1, V2, V3, V4, V5, V6, V7, V8, V9 and V10) and two from goats (C11 and $\mathrm{C} 12)$. Then, three different lots from every brand were selected.

\section{Proximate composition}

According to the AOAC methods (Cunniff), the moisture and ash contents were determined through heating in a laboratory oven at $105^{\circ} \mathrm{C}$ and incineration in a muffle furnace at $600{ }^{\circ} \mathrm{C}$, respectively. ${ }^{14}$ The protein contents were analyzed through the semi-micro Kjeldahl method and the total carbohydrate content was estimated through mass difference.

For the total lipid determination, samples were prepared following the instructions from the product packages ( $26 \mathrm{~g}$ of powder for $200 \mathrm{~mL}$ of water) and submitted to extraction with a mixture of chloroform, methanol and water, according to Folch et al. ${ }^{15}$

\section{Fatty acids analysis}

The fatty acid esterifications were done through the Hartman and Lago method. ${ }^{16}$ The synthesized fatty acid methyl esters (FAMEs) were separated in a gas chromatograph (Shimadzu 14A, Japan), equipped with a flame ionization detector (FID) and capillary column of fused silica CP-Sil88, with length of $100 \mathrm{~m}, 0.25 \mathrm{~mm}$ of internal diameter and $0.25 \mu \mathrm{m}$ of cyanopropyl polysiloxane.

The employed chromatographic conditions for the FA separations were: injection point and detector temperatures of 220 and $240{ }^{\circ} \mathrm{C}$, respectively, whereas the initial column temperature was programmed to $65^{\circ} \mathrm{C}$ for $4 \mathrm{~min}$, increased from 65 to $170{ }^{\circ} \mathrm{C}$ with a rate of $20^{\circ} \mathrm{C} \mathrm{min}^{-1}$ during $20 \mathrm{~min}$ and once again increased from 170 to $235^{\circ} \mathrm{C}$ at $6{ }^{\circ} \mathrm{C} \mathrm{min}^{-1}$, for $8 \mathrm{~min}$. The gas (White Martins brand) flow rates used were: $1.4 \mathrm{~mL} \mathrm{~min}^{-1}$ (carrier gas- $\mathrm{H}_{2}$ ); $30 \mathrm{~mL} \mathrm{~min}{ }^{-1}$ (make-up gas- $\mathrm{N}_{2}$ ), 30 and $300 \mathrm{~mL} \mathrm{~min}^{-1}$ for the flame gases $\mathrm{H}_{2}$ and synthetic air, respectively. The sample split ratio was $1 / 100$. 
Identification of fatty acids

The fatty acid identification was made through the comparison of relative retention times of the sample peaks with a mixture of FAME standards and methyl esters containing linoleic acid geometric isomers c9,t11 and t10,c12 (189-19 and O5632 Sigma, USA) and standard spiking along the sample. The absolute quantification (mg FA per $\mathrm{g}$ of total lipids) was achieved employing methyl tricosanoate (IS, 23:0) as internal standard (IS), as described by Joseph and Ackman. ${ }^{17}$ To obtain the FA concentration values, a theoretical FID correction factor, according to Visentainer, ${ }^{18}$ was used. FA contents were calculated in FA mg per $\mathrm{g}$ of total lipids employing the equation 1 ,

$\mathrm{FA}\left(\mathrm{mg} \mathrm{g}^{-1}\right)=\frac{\mathrm{A}_{\mathrm{X}} \mathrm{W}_{\mathrm{IS}} \mathrm{CF}_{\mathrm{X}}}{\mathrm{A}_{\mathrm{IS}} \mathrm{W}_{\mathrm{X}} \mathrm{CF}_{\mathrm{AE}}}$

where $A_{X}$ is the peak area (of a respective FA), $A_{I S}$ is the internal standard peak area, $\mathrm{W}_{\mathrm{IS}}$ is the IS weight $(\mathrm{mg})$ added to the sample, $\mathrm{W}_{\mathrm{X}}$ is the sample weight $(\mathrm{mg}), \mathrm{CF}_{\mathrm{X}}$ is the theoretical correction factor and $\mathrm{CF}_{\mathrm{AE}}$ is the conversion factor necessary to express the results in FA instead of methyl esters. The injections were done in triplicates with volumes of $2 \mathrm{~mL}$.

\section{Statistical analysis}

For the statistical analysis, the results were submitted to variance (ANOVA) analysis and, in case of differences, they were compared through the Tukey's test with $5.0 \%$ of significance on the SAS software with statistical package. ${ }^{19}$

\section{Results and Discussion}

Table 1 shows the results of the physicochemical analysis from the samples. According to the Ministry of Agriculture, Livestock and Food Supplies (MAPA) ordinance No. 146/1996, ${ }^{20}$ whole powdered milks may contain a maximum moisture content of $3.5 \%$ and a total lipid amount equal or greater than $26 \%$.

Three samples showed moisture values that significantly extrapolated their limit. However, its alert limit is $7 \%$. Regarding ash percentages, powdered goat milks showed greater values due to their high mineral concentrations, according to Trancoso et al. ${ }^{21}$

In the spray drying process, milk proteins, fats and lactose migrate to the surface, which facilitates the sample oxidation and the denaturation of its proteins. These reactions are more intense when the temperature is increased. ${ }^{22,23}$ However, all the values obtained from the proximate composition analyses showed that all the products possess an adequate nutritional quality.

Through the chromatographic analysis, 31 fatty acids were detected among the total lipids of the analyzed milks. The predominant sample FAs were the myristic (14:0), palmitic (16:0), stearic (18:0) and oleic acid (18:1n-9) (Table 2). From the four FAs that were detected, only the oleic acid is a monounsaturated acid, while the rest is saturated.

It is worth mentioning that oleic and stearic acids are CLA precursors. They suffer action of desaturase enzymes in the mammary glands, being converted to vaccenic acid 18:1(t11) that is converted to CLA afterwards. ${ }^{24}$ Another reaction route occurs through the action of bacteria (Butyrinvibrio fibrisolvens) presents on the rumen that converts LA to CLA. ${ }^{25}$

Table 1. Proximate composition (g per $100 \mathrm{~g}$ of food) of whole cow and goat powdered milks, Maringá (PR)

\begin{tabular}{|c|c|c|c|c|c|}
\hline Sample & Moisture & Ash & Protein & Lipid & Carbohydrate \\
\hline $\mathrm{V} 1$ & $3.66^{\mathrm{cd}} \pm 0.02$ & $6.07^{\mathrm{d}} \pm 0.04$ & $25.91^{\text {b.c.d }} \pm 0.82$ & $26.68^{b} \pm 0.12$ & $37.69^{\text {b.c }} \pm 0.74$ \\
\hline $\mathrm{V} 2$ & $3.17^{\mathrm{fg} g} \pm 0.02$ & $5.96^{\mathrm{d} . \mathrm{e}} \pm 0.02$ & $24.73^{\mathrm{d}} \pm 0.39$ & $26.62^{\mathrm{b}} \pm 0.02$ & $39.52^{\mathrm{a} . \mathrm{b}} \pm 0.39$ \\
\hline V3 & $3.22^{e . f} \pm 0.01$ & $5.89^{\mathrm{e} . \mathrm{f}} \pm 0.01$ & $27.39^{\mathrm{a}} \pm 0.43$ & $26.32^{\text {b.c.d }} \pm 0.24$ & $37.17^{\text {c.d }} \pm 0.60$ \\
\hline V4 & $3.83^{c} \pm 0.08$ & $5.79^{\mathrm{fg} g} \pm 0.02$ & $26.06^{\text {a.b.c.d }} \pm 0.12$ & $28.50^{\mathrm{a}} \pm 0.54$ & $35.81^{\text {d.e }} \pm 0.51$ \\
\hline V5 & $3.42^{\text {d.e.f }} \pm 0.08$ & $5.82^{\mathrm{f} . \mathrm{g}} \pm 0.06$ & $25.91^{\text {b.c.d }} \pm 0.20$ & $26.85^{\mathrm{b}} \pm 0.33$ & $38.00^{\text {a.b.c }} \pm 0.55$ \\
\hline V6 & $3.48^{\text {d.e }} \pm 0.04$ & $5.74^{\mathrm{f}} \pm 0.04$ & $26.42^{\text {a.b.c }} \pm 0.73$ & $26.12^{\text {b.c.d }} \pm 0.67$ & $38.24^{\text {a.b.c }} \pm 0.87$ \\
\hline V7 & $4.66^{a} \pm 0.14$ & $5.79^{\mathrm{f} . \mathrm{g}} \pm 0.08$ & $24.82^{\mathrm{d}} \pm 0.53$ & $26.63^{\mathrm{b}} \pm 0.06$ & $38.10^{\text {a.b.c }} \pm 0.45$ \\
\hline V8 & $2.76^{\mathrm{h}} \pm 0.04$ & $5.97^{\mathrm{d} . \mathrm{e}} \pm 0.02$ & $25.07^{\mathrm{c.d}} \pm 0.65$ & $26.37^{\text {b.c }} \pm 0.68$ & $39.83^{\mathrm{a}} \pm 0.74$ \\
\hline V9 & $2.92^{\mathrm{g} . \mathrm{h}} \pm 0.08$ & $6.24^{\mathrm{c}} \pm 0.07$ & $26.78^{\mathrm{a} . \mathrm{b}} \pm 0.43$ & $25.07^{\mathrm{d}} \pm 0.73$ & $38.99^{\text {a.b.c }} \pm 0.93$ \\
\hline V10 & $3.42^{\text {d.e.f }} \pm 0.23$ & $5.82^{\mathrm{f} \cdot \mathrm{g}} \pm 0.05$ & $24.65^{\mathrm{d}} \pm 0.17$ & $26.84^{\mathrm{b}} \pm 0.09$ & $39.27^{\mathrm{a} \cdot \mathrm{b}} \pm 0.24$ \\
\hline $\mathrm{C} 11$ & $3.66^{\mathrm{cd}} \pm 0.04$ & $6.48^{\mathrm{b}} \pm 0.00$ & $24.98^{\mathrm{d}} \pm 0.31$ & $25.14^{\mathrm{c} . \mathrm{d}} \pm 0.42$ & $39.73^{a} \pm 0.65$ \\
\hline $\mathrm{C} 12$ & $4.17^{\mathrm{b}} \pm 0.02$ & $7.35^{\mathrm{a}} \pm 0.06$ & $25.27^{\mathrm{c} . \mathrm{d}} \pm 0.42$ & $28.66^{a} \pm 0.47$ & $34.56^{\mathrm{e}} \pm 0.52$ \\
\hline
\end{tabular}

Means that are followed by different letters in a same column differ significantly according to Tukey's test (5\%). 
Table 2. Main fatty acids (mg per $\mathrm{g}$ of total lipids) of whole cow and goat powdered milks, Maringá (PR)

\begin{tabular}{|c|c|c|c|c|c|c|}
\hline Fatty acid & $\mathrm{V} 1$ & $\mathrm{~V} 2$ & V3 & V4 & V5 & V6 \\
\hline $4: 0$ & $24.81^{\mathrm{a}, \mathrm{b}} \pm 0.65$ & $16.77^{\mathrm{d}, \mathrm{e}} \pm 1.31$ & $15.19^{\mathrm{e}, \mathrm{f}} \pm 1.01$ & $28.46^{\mathrm{a}} \pm 2.63$ & $19.16^{\mathrm{cd}} \pm 0.29$ & $16.70^{\mathrm{d}, \mathrm{e}} \pm 0.65$ \\
\hline $6: 0$ & $22.40^{\mathrm{a}, \mathrm{b}} \pm 0.35$ & $17.46^{\mathrm{c}, \mathrm{d}, \mathrm{e}} \pm 1.50$ & $15.71^{\mathrm{e}} \pm 0.86$ & $21.99^{\mathrm{a}, \mathrm{b}} \pm 1.97$ & $19.00^{\mathrm{b}, \mathrm{c}, \mathrm{d}, \mathrm{e}} \pm 0.29$ & $17.88^{\mathrm{c}, \mathrm{d}, \mathrm{e}} \pm 0.65$ \\
\hline $8: 0$ & $13.81^{\mathrm{c}} \pm 0.14$ & $11.99^{\mathrm{c}, \mathrm{d}} \pm 1.27$ & $10.89^{\mathrm{e}} \pm 0.57$ & $12.85^{\mathrm{c}, \mathrm{d}} \pm 0.53$ & $12.72^{\mathrm{c}, \mathrm{d}} \pm 0.22$ & $13.01^{\mathrm{c}, \mathrm{d}} \pm 0.43$ \\
\hline $10: 0$ & $28.89 \pm 0.26$ & $26.84 \pm 3.30$ & $23.21 \pm 1.25$ & $26.61 \pm 0.56$ & $26.72 \pm 0.75$ & $29.66 \pm 0.68$ \\
\hline $10: 1 n-1$ & $2.85^{\mathrm{c}, \mathrm{d}} \pm 0.04$ & $2.68^{\mathrm{d}} \pm 0.24$ & $3.00^{\mathrm{b}, \mathrm{c}} \pm 0.11$ & $2.72^{\mathrm{d}} \pm 0.09$ & $3.17^{\mathrm{a}, \mathrm{b}} \pm 0.08$ & $2.91^{\mathrm{b}, c, d} \pm 0.11$ \\
\hline 11:0 & $0.41^{\mathrm{c}, \mathrm{d}} \pm 0.03$ & $0.23^{\mathrm{e}} \pm 0.01$ & $0.25^{\mathrm{e}} \pm 0.03$ & $0.40^{\mathrm{c}, \mathrm{d}} \pm 0.02$ & $0.31^{\mathrm{d}, \mathrm{e}} \pm 0.01$ & $0.45^{\mathrm{c}} \pm 0.04$ \\
\hline $12: 0$ & $30.94^{\mathrm{b}, \mathrm{c}, \mathrm{d}, \mathrm{e}} \pm 0.34$ & $29.68^{\mathrm{cdd}, \mathrm{de}, \mathrm{f}} \pm 2.58$ & $27.18^{\mathrm{f}} \pm 1.18$ & $28.35^{\mathrm{d}, \mathrm{e}, \mathrm{f}} \pm 0.51$ & $29.92^{\mathrm{c}, \mathrm{d}, \mathrm{e}, \mathrm{f}} \pm 0.95$ & $32.85^{\mathrm{b}, \mathrm{c}} \pm 0.44$ \\
\hline $13: 0$ & $0.80^{\mathrm{d}} \pm 0.01$ & $0.75^{\mathrm{d}} \pm 0.06$ & $0.84^{\mathrm{c}, \mathrm{d}} \pm 0.09$ & $1.20^{\mathrm{a}} \pm 0.06$ & $0.79^{\mathrm{d}} \pm 0.02$ & $0.92^{b, c} \pm 0.03$ \\
\hline $14: 0$ & $106.36^{\mathrm{b}, \mathrm{c}, \mathrm{d}, \mathrm{e}} \pm 0.58$ & $107.78^{\mathrm{a}, \mathrm{b}, \mathrm{c}, \mathrm{d}} \pm 6.53$ & $101.48^{\mathrm{d}, \mathrm{ef}} \pm 3.89$ & $99.69^{e . f} \pm 0.74$ & $112.47^{\mathrm{b}} \pm 2.24$ & $109.69^{\mathrm{a}, \mathrm{b}, \mathrm{c}} \pm 0.73$ \\
\hline $14: 1 n-11$ & $10.94^{\mathrm{d}} \pm 0.06$ & $3.26^{g} \pm 0.15$ & $4.77^{\mathrm{f}} \pm 0.21$ & $10.33^{\mathrm{e}} \pm 0.13$ & $14.72^{a} \pm 0.32$ & $2.88^{\mathrm{g}} \pm 0.057$ \\
\hline $14: 1 \mathrm{n}-9$ & $0.25^{\mathrm{g}} \pm 0.04$ & $8.03^{d} \pm 0.27$ & $10.40^{\mathrm{a}} \pm 0.29$ & $0.27^{\mathrm{g}} \pm 0.02$ & $0.25^{\mathrm{g}} \pm 0.02$ & $8.88^{c} \pm 0.14$ \\
\hline $14: 1 n-7$ & $4.69^{\mathrm{e}} \pm 0.04$ & $6.02^{c} \pm 0.15$ & $7.81^{\mathrm{a}} \pm 0.23$ & $5.17^{\mathrm{d}} \pm 0.06$ & $6.43^{b} \pm 0.11$ & $6.11^{\mathrm{c}} \pm 0.04$ \\
\hline $15: 0$ & $9.26^{\mathrm{f}} \pm 0.04$ & $10.28^{\mathrm{e}} \pm 0.28$ & $13.00^{\mathrm{a}} \pm 0.33$ & $11.28^{b} \pm 0.16$ & $10.27^{\mathrm{e}} \pm 0.14$ & $10.80^{\mathrm{c}, \mathrm{d}} \pm 0.13$ \\
\hline $15: 1 \mathrm{n}-7$ & $2.38^{\mathrm{f}} \pm 0.03$ & $2.67^{\mathrm{c}, \mathrm{de} e} \pm 0.15$ & $3.27^{\mathrm{a}} \pm 0.10$ & $2.62^{\text {d.e }} \pm 0.05$ & $2.82^{\mathrm{c}} \pm 0.03$ & $2.52^{\mathrm{e}, \mathrm{f}} \pm 0.06$ \\
\hline $16: 0$ & $282.27^{\mathrm{c}} \pm 1.10$ & $281.88^{c} \pm 6.96$ & $266.29^{\mathrm{d}} \pm 5.43$ & $276.05^{\mathrm{c}} \pm 2.88$ & $296.46^{a \cdot b} \pm 0.78$ & $260.38^{d} \pm 1.25$ \\
\hline $16: 1 n-11$ & $2.18^{\mathrm{c}, \mathrm{d}, \mathrm{e}, \mathrm{f}} \pm 0.01$ & $2.33^{\mathrm{c}, \mathrm{d}} \pm 0.04$ & $2.95^{\mathrm{b}} \pm 0.09$ & $1.99^{\mathrm{f}} \pm 0.10$ & $2.05^{\mathrm{e}, \mathrm{f}} \pm 0.04$ & $2.37^{\mathrm{c}, \mathrm{d}} \pm 0.12$ \\
\hline $16: 1 n-9$ & $12.64^{\mathrm{f}} \pm 0.16$ & $14.12^{\mathrm{d}} \pm 0.26$ & $16.87^{\mathrm{a}} \pm 0.37$ & $10.47^{\mathrm{h}} \pm 0.05$ & $12.19^{f} \pm 0.04$ & $14.86^{c} \pm 0.17$ \\
\hline $16: 1 n-7$ & $3.73^{\mathrm{f}, \mathrm{g}} \pm 0.08$ & $4.99^{\mathrm{c}, \mathrm{d}} \pm 0.22$ & $5.83^{\mathrm{a}} \pm 0.11$ & $3.56^{g} \pm 0.01$ & $3.99^{\mathrm{e}, \mathrm{f}} \pm 0.08$ & $5.13^{b, c} \pm 0.08$ \\
\hline $17: 0$ & $5.06^{g} \pm 0.02$ & $5.97^{\mathrm{c}, \mathrm{d}} \pm 0.12$ & $7.17^{\mathrm{a}} \pm 0.16$ & $7.13^{\mathrm{a}} \pm 0.19$ & $6.17^{\mathrm{c}} \pm 0.08$ & $5.38^{\mathrm{e}, \mathrm{f}} \pm 0.05$ \\
\hline $17: 1 \mathrm{n}-7$ & $2.11^{\mathrm{de}, \mathrm{f} f} \pm 0.05$ & $2.16^{\mathrm{c}, \mathrm{d}, \mathrm{e}, \mathrm{f}} \pm 0.07$ & $3.26^{a} \pm 0.09$ & $2.36^{b, c} \pm 0.08$ & $2.30^{\mathrm{b}, \mathrm{c}, \mathrm{d}, \mathrm{e}} \pm 0.04$ & $1.97^{\mathrm{f}, \mathrm{g}} \pm 0.08$ \\
\hline $18: 0$ & $100.01^{\mathrm{e}} \pm 0.77$ & $107.76^{\mathrm{b}, \mathrm{c}, \mathrm{d}} \pm 2.23$ & $110.28^{\mathrm{b}} \pm 0.81$ & $107.92^{b, c, d} \pm 1.62$ & $102.87^{\mathrm{d}, \mathrm{e}} \pm 1.59$ & $108.57^{\mathrm{b}, c, d} \pm 1.03$ \\
\hline $18: 1(\mathrm{t} 11)$ & $13.40^{\mathrm{e}} \pm 0.93$ & $19.22^{\mathrm{b}, \mathrm{c}} \pm 1.15$ & $20.85^{\mathrm{a}, \mathrm{b}} \pm 0.98$ & $21.48^{\mathrm{a}} \pm 1.17$ & $16.48^{\mathrm{d}} \pm 0.56$ & $21.47^{\mathrm{a}} \pm 0.22$ \\
\hline $18: 1 \mathrm{n}-9$ & $197.94^{\mathrm{b}, \mathrm{d}, \mathrm{d}} \pm 0.67$ & $188.94^{\mathrm{d}, e, \mathrm{f}} \pm 10.04$ & $217.22^{\mathrm{a}} \pm 6.07$ & $196.72^{\mathrm{b}, \mathrm{c}, \mathrm{d}, \mathrm{e}} \pm 2.64$ & $185.23^{\mathrm{e}, \mathrm{f}} \pm 2.71$ & $195.21^{b, c, d, e, f} \pm 1.58$ \\
\hline $18: 1 n-7$ & $4.20^{\mathrm{b}} \pm 0.09$ & $3.83^{\mathrm{c}} \pm 0.15$ & $3.78^{c} \pm 0.06$ & $4.67^{\mathrm{a}} \pm 0.17$ & $3.27^{\mathrm{d}} \pm 0.06$ & $3.88^{c} \pm 0.14$ \\
\hline $18: 2(\mathrm{t} 12)$ & $1.04^{\mathrm{d}, \mathrm{e}} \pm 0.07$ & $0.84^{\mathrm{e}, \mathrm{fg} \mathrm{g}} \pm 0.09$ & $0.69^{\mathrm{f}, \mathrm{g}} \pm 0.06$ & $1.66^{\mathrm{b}} \pm 0.06$ & $0.66^{g} \pm 0.07$ & $1.73^{\mathrm{b}} \pm 0.18$ \\
\hline $18: 2 n-6$ & $19.57^{\mathrm{b}, \mathrm{c}} \pm 0.67$ & $30.58^{a} \pm 3.36$ & $14.57^{\mathrm{d}, \mathrm{e}, \mathrm{f}} \pm 1.95$ & $21.25^{\mathrm{b}} \pm 0.57$ & $12.95^{\mathrm{e}, \mathrm{f}} \pm 0.58$ & $22.50^{\mathrm{b}} \pm 0.26$ \\
\hline $18: 3 n-6$ & $0.42^{g} \pm 0.02$ & $0.87^{\mathrm{b}} \pm 0.06$ & $1.25^{\mathrm{a}} \pm 0.11$ & $0.53^{\mathrm{f} .} \pm 0.03$ & $0.76^{\mathrm{b}, \mathrm{c}, \mathrm{d}} \pm 0.06$ & $0.69^{\mathrm{c}, \mathrm{d}, \mathrm{e}} \pm 0.08$ \\
\hline $18: 3 n-3$ & $4.64^{\mathrm{d}} \pm 0.02$ & $5.17^{\mathrm{c}} \pm 0.28$ & $4.25^{\mathrm{e}} \pm 0.14$ & $7.65^{\mathrm{a}} \pm 0.07$ & $3.45^{\mathrm{f}} \pm 0.04$ & $6.16^{\mathrm{b}} \pm 0.19$ \\
\hline $20: 0$ & $1.21^{\mathrm{f}} \pm 0.01$ & $1.66^{\mathrm{b} \cdot \mathrm{c}} \pm 0.14$ & $1.58^{\mathrm{b}, \mathrm{c}, \mathrm{d}} \pm 0.10$ & $1.42^{\mathrm{d}, \mathrm{e}, \mathrm{f}} \pm 0.02$ & $1.38^{\mathrm{d}, \mathrm{e}, \mathrm{f}} \pm 0.04$ & $1.34^{\mathrm{e}, \mathrm{f}} \pm 0.05$ \\
\hline $18: 2(\mathrm{c} 9, \mathrm{t} 11)$ & $7.37^{\mathrm{d}} \pm 0.06$ & $7.83^{\mathrm{c}, \mathrm{d}} \pm 0.20$ & $10.11^{\mathrm{a}} \pm 0.26$ & $10.10^{\mathrm{a}} \pm 0.12$ & $8.05^{\mathrm{b}, \mathrm{c}} \pm 0.019$ & $9.78^{\mathrm{a}} \pm 0.11$ \\
\hline \multirow[t]{2}{*}{$18: 2(\mathrm{t} 10, \mathrm{c} 12)$} & $0.81^{\mathrm{e}, \mathrm{f}} \pm 0.02$ & $1.36^{\mathrm{a}, \mathrm{b}} \pm 0.12$ & $1.49^{\mathrm{a}} \pm 0.03$ & $1.02^{\mathrm{c.d}} \pm 0.03$ & $1.01^{\mathrm{c}, \mathrm{d}} \pm 0.03$ & $1.29^{\mathrm{b}} \pm 0.04$ \\
\hline & V7 & V8 & V9 & V10 & $\mathrm{C} 11$ & $\mathrm{C} 12$ \\
\hline $4: 0$ & $22.21^{\mathrm{b}, \mathrm{c}} \pm 2.83$ & $15.01^{\mathrm{e}, \mathrm{f}} \pm 1.61$ & $23.45^{\mathrm{b}} \pm 1.36$ & $23.10^{\mathrm{b}} \pm 1.77$ & $18.32^{\mathrm{d}, \mathrm{e}} \pm 0.41$ & $12.01^{\mathrm{f}} \pm 1.30$ \\
\hline $6: 0$ & $20.57^{\mathrm{b}, \mathrm{c}} \pm 1.77$ & $16.61^{\mathrm{d}, \mathrm{e}} \pm 0.43$ & $22.15^{\mathrm{a}, \mathrm{b}} \pm 0.59$ & $19.83^{\mathrm{b}, \mathrm{c}, \mathrm{d}} \pm 2.38$ & $25.28^{a} \pm 2.74$ & $19.69^{\mathrm{b}, \mathrm{c}, \mathrm{d}} \pm 1.55$ \\
\hline $8: 0$ & $13.05^{\mathrm{c}, \mathrm{d}} \pm 0.44$ & $11.89^{\mathrm{c}, \mathrm{d}} \pm 0.45$ & $14.15^{\mathrm{c}} \pm 0.29$ & $12.05^{\mathrm{c}, \mathrm{d}} \pm 0.93$ & $30.71^{\mathrm{a}} \pm 2.93$ & $25.17^{\mathrm{b}} \pm 1.35$ \\
\hline $10: 0$ & $28.06 \pm 0.25$ & $27.12 \pm 0.91$ & $30.71 \pm 1.02$ & $25.15 \pm 1.68$ & $95.96^{a} \pm 8.71$ & $88.26^{\mathrm{b}} \pm 4.23$ \\
\hline $10: 1 n-1$ & $2.80^{c . d} \pm 0.07$ & $2.89^{\mathrm{c}, \mathrm{d}} \pm 0.06$ & $3.37^{\mathrm{a}} \pm 0.07$ & $2.84^{\mathrm{c}, \mathrm{d}} \pm 0.19$ & $0.30^{\mathrm{e}} \pm 0.03$ & $0.37^{\mathrm{e}} \pm 0.02$ \\
\hline 11:0 & $0.33^{\mathrm{d}, \mathrm{e}} \pm 0.01$ & $0.39^{\mathrm{c}, \mathrm{d}} \pm 0.03$ & $0.50^{c} \pm 0.02$ & $0.31^{\mathrm{d}, \mathrm{e}} \pm 0.02$ & $2.34^{\mathrm{a}} \pm 0.11$ & $1.87^{\mathrm{b}} \pm 0.07$ \\
\hline $12: 0$ & $30.26^{\mathrm{c}, \mathrm{d}, \mathrm{e}, \mathrm{f}} \pm 0.35$ & $31.69^{\mathrm{b}, \mathrm{c}, \mathrm{d}} \pm 0.94$ & $34.01^{\mathrm{a}, \mathrm{b}} \pm 1.28$ & $28.08^{e, f} \pm 1.71$ & $36.35^{\mathrm{a}} \pm 2.79$ & $36.72^{\mathrm{a}} \pm 0.97$ \\
\hline 13:0 & $0.75^{\mathrm{d}} \pm 0.01$ & $0.92^{\mathrm{b}, \mathrm{c}} \pm 0.02$ & $0.96^{\mathrm{b}} \pm 0.03$ & $0.77^{\mathrm{d}} \pm 0.05$ & $0.73^{\mathrm{d}} \pm 0.06$ & $0.81^{\mathrm{c}, \mathrm{d}} \pm 0.05$ \\
\hline $14: 0$ & $106.93^{\mathrm{b}, \mathrm{c}, \mathrm{d}, \mathrm{e}} \pm 1.72$ & $109.31^{\mathrm{a}, \mathrm{b}, \mathrm{c}} \pm 1.74$ & $115.27^{\mathrm{a}} \pm 3.47$ & $103.05^{\mathrm{c}, \mathrm{d}, \mathrm{e}, \mathrm{f}} \pm 5.11$ & $86.25^{\mathrm{g}} \pm 1.33$ & $96.31^{\mathrm{f}} \pm 0.76$ \\
\hline $14: 1 n-11$ & $11.15^{\mathrm{c}, \mathrm{d}} \pm 0.15$ & $3.40^{g} \pm 0.08$ & $13.33^{b} \pm 0.35$ & $11.65^{c} \pm 0.46$ & $1.82^{\mathrm{h}} \pm 0.06$ & $2.04^{\mathrm{h}} \pm 0.04$ \\
\hline $14: 1 n-9$ & $0.22^{\mathrm{g}} \pm 0.02$ & $9.44^{\mathrm{b}} \pm 0.15$ & $0.35^{g} \pm 0.02$ & $0.23^{\mathrm{g}} \pm 0.02$ & $1.72^{\mathrm{e}} \pm 0.02$ & $1.25^{\mathrm{f}} \pm 0.04$ \\
\hline $14: 1 n-7$ & $5.32^{\mathrm{d}} \pm 0.06$ & $6.07^{c} \pm 0.09$ & $5.40^{\mathrm{d}} \pm 0.10$ & $5.35^{\mathrm{d}} \pm 0.14$ & $3.81^{\mathrm{g}} \pm 0.13$ & $4.39^{\mathrm{f}} \pm 0.11$ \\
\hline
\end{tabular}


Table 2. continuation

\begin{tabular}{|c|c|c|c|c|c|c|}
\hline Fatty acid & V7 & V8 & V9 & V10 & C11 & $\mathrm{C} 12$ \\
\hline $15: 0$ & $10.36^{\mathrm{d}, \mathrm{e}} \pm 0.15$ & $10.58^{\mathrm{c}, \mathrm{d}, \mathrm{e}} \pm 0.21$ & $10.66^{\mathrm{c}, \mathrm{d}, \mathrm{e}} \pm 0.15$ & $10.99^{\mathrm{b}, \mathrm{c}} \pm 0.22$ & $7.26^{\mathrm{h}} \pm 0.06$ & $8.05^{g} \pm 0.08$ \\
\hline $15: 1 n-7$ & $2.83^{c} \pm 0.02$ & $2.57^{\mathrm{d}, \mathrm{e}} \pm 0.01$ & $2.73^{\mathrm{c}, \mathrm{d}} \pm 0.03$ & $3.07^{\mathrm{b}} \pm 0.03$ & $1.84^{\mathrm{g}} \pm 0.14$ & $2.00^{\mathrm{g}} \pm 0.09$ \\
\hline $16: 0$ & $277.95^{\mathrm{c}} \pm 2.49$ & $296.02^{\mathrm{b}} \pm 4.22$ & $305.15^{\mathrm{a}} \pm 1.22$ & $283.72^{c} \pm 1.55$ & $247.24^{\mathrm{e}} \pm 5.81$ & $257.94^{\mathrm{d}} \pm 1.22$ \\
\hline $16: 1 n-11$ & $2.16^{\mathrm{d}, \mathrm{e}, \mathrm{f}} \pm 0.05$ & $2.38^{c} \pm 0.15$ & $2.04^{\mathrm{e}, \mathrm{f}} \pm 0.03$ & $2.24^{\mathrm{c}, \mathrm{d}, \mathrm{e}} \pm 0.02$ & $2.89^{\mathrm{b}} \pm 0.14$ & $3.45^{\mathrm{a}} \pm 0.10$ \\
\hline $16: 1 n-9$ & $11.23^{\mathrm{g}} \pm 0.13$ & $15.75^{\mathrm{b}} \pm 0.10$ & $13.33^{\mathrm{e}} \pm 0.07$ & $11.65^{\mathrm{g}} \pm 0.07$ & $8.37^{\mathrm{i}} \pm 0.21$ & $8.27^{\mathrm{i}} \pm 0.20$ \\
\hline $16: 1 n-7$ & $4.17^{\mathrm{e}} \pm 0.06$ & $5.30^{\mathrm{b}} \pm 0.07$ & $4.13^{\mathrm{e}} \pm 0.02$ & $3.71^{\mathrm{f}, \mathrm{g}} \pm 0.11$ & $3.89^{\mathrm{e}, \mathrm{f}} \pm 0.22$ & $4.82^{\mathrm{d}} \pm 0.15$ \\
\hline $17: 0$ & $6.25^{c} \pm 0.10$ & $5.70^{\mathrm{d}, \mathrm{e}} \pm 0.04$ & $5.52^{\mathrm{e}, \mathrm{f}} \pm 0.08$ & $6.57^{b} \pm 0.05$ & $4.48^{\mathrm{h}} \pm 0.19$ & $5.30^{\mathrm{f}, \mathrm{g}} \pm 0.24$ \\
\hline $17: 1 n-7$ & $2.27^{\mathrm{b}, \mathrm{c}, \mathrm{d}, \mathrm{e}} \pm 0.03$ & $2.35^{\mathrm{b}, \mathrm{c}} \pm 0.04$ & $2.33^{\mathrm{b}, \mathrm{c}, \mathrm{d}} \pm 0.03$ & $2.40^{\mathrm{b}} \pm 0.06$ & $1.74^{g} \pm 0.23$ & $2.06^{\mathrm{e} . \mathrm{f}} \pm 0.13$ \\
\hline 18:0 & $116.88^{\mathrm{a}} \pm 0.42$ & $104.51^{\mathrm{c}, \mathrm{d}, \mathrm{e}} \pm 0.54$ & $93.62^{\mathrm{f}} \pm 2.14$ & $109.70^{\mathrm{b}, \mathrm{c}} \pm 4.06$ & $107.80^{\mathrm{b}, \mathrm{c}, \mathrm{d}} \pm 4.67$ & $103.32^{\mathrm{d}, \mathrm{e}} \pm 2.93$ \\
\hline $18: 1(\mathrm{t} 11)$ & $17.95^{\mathrm{c}, \mathrm{d}} \pm 0.40$ & $14.33^{\mathrm{e}} \pm 0.20$ & $13.23^{\mathrm{e}, \mathrm{f}} \pm 0.57$ & $18.42^{\mathrm{c}, \mathrm{d}} \pm 1.23$ & $14.50^{\mathrm{e}} \pm 0.62$ & $11.38^{\mathrm{f}} \pm 0.20$ \\
\hline $18: 1 n-9$ & $202.62^{\mathrm{b}, \mathrm{c}} \pm 1.50$ & $190.76^{\mathrm{c}, \mathrm{d}, \mathrm{e}, \mathrm{f}} \pm 5.22$ & $183.18^{f} \pm 3.62$ & $206.11^{\mathrm{a}, \mathrm{b}} \pm 7.51$ & $186.70^{\mathrm{d}, \mathrm{e}, \mathrm{f}} \pm 5.79$ & $189.15^{\mathrm{d}, \mathrm{e}, \mathrm{f}} \pm 4.56$ \\
\hline $18: 1 n-7$ & $4.27^{\mathrm{b}} \pm 0.04$ & $3.71^{\mathrm{c}} \pm 0.09$ & $3.73^{c} \pm 0.14$ & $4.40^{\mathrm{a}, \mathrm{b}} \pm 0.16$ & $2.29^{\mathrm{e}} \pm 0.18$ & $3.32^{\mathrm{d}} \pm 0.06$ \\
\hline $18: 2(\mathrm{t} 12)$ & $0.91^{\mathrm{d}, \mathrm{e}, \mathrm{f}} \pm 0.02$ & $1.34^{\mathrm{c}} \pm 0.04$ & $1.12^{\mathrm{c}, \mathrm{d}} \pm 0.09$ & $1.09^{\mathrm{d}} \pm 0.06$ & $2.68^{\mathrm{a}} \pm 0.15$ & $1.62^{b} \pm 0.13$ \\
\hline $18: 2 n-6$ & $17.10^{\mathrm{c}, \mathrm{d}} \pm 0.63$ & $15.27^{\mathrm{d}, \mathrm{e}, \mathrm{f}} \pm 1.65$ & $12.20^{\mathrm{f}} \pm 0.42$ & $15.54^{\mathrm{d}, \mathrm{e}} \pm 0.57$ & $22.00^{\mathrm{b}} \pm 0.42$ & $28.80^{\mathrm{a}} \pm 0.74$ \\
\hline $18: 3 n-6$ & $0.58^{\mathrm{e}, \mathrm{f}} \pm 0.01$ & $0.80^{\mathrm{b}, \mathrm{c}} \pm 0.07$ & $0.61^{\mathrm{d}, \mathrm{e}, \mathrm{f}} \pm 0.02$ & $0.73^{\mathrm{b}, \mathrm{c}, \mathrm{d}} \pm 0.07$ & $0.39^{\mathrm{g}} \pm 0.05$ & $0.49^{\mathrm{f}, \mathrm{g}} \pm 0.05$ \\
\hline $18: 3 n-3$ & $5.36^{\mathrm{c}} \pm 0.08$ & $2.70^{\mathrm{h}} \pm 0.06$ & $3.05^{\mathrm{g}} \pm 0.09$ & $4.65^{\mathrm{d}} \pm 0.15$ & $1.77^{\mathrm{i}} \pm 0.15$ & $2.59^{\mathrm{h}} \pm 0.07$ \\
\hline $20: 0$ & $1.44^{\mathrm{c}, \mathrm{d}, \mathrm{e}} \pm 0.01$ & $1.33^{\mathrm{e}, \mathrm{f}} \pm 0.06$ & $1.21^{\mathrm{f}} \pm 0.06$ & $1.46^{\mathrm{c}, \mathrm{d}, \mathrm{e}} \pm 0.09$ & $2.00^{\mathrm{a}} \pm 0.16$ & $1.72^{b} \pm 0.16$ \\
\hline $18: 2(\mathrm{c} 9, \mathrm{t} 11)$ & $8.57^{b} \pm 0.14$ & $6.69^{e} \pm 0.13$ & $7.29^{\mathrm{d}} \pm 0.21$ & $9.77^{\mathrm{a}} \pm 0.47$ & $7.83^{\mathrm{c}, \mathrm{d}} \pm 0.31$ & $6.08^{\mathrm{f}} \pm 0.21$ \\
\hline $18: 2(\mathrm{t} 10, \mathrm{c} 12)$ & $0.90^{\mathrm{d}, \mathrm{e}, \mathrm{f}} \pm 0.01$ & $1.08^{c} \pm 0.02$ & $0.92^{\mathrm{d}, \mathrm{e}} \pm 0.02$ & $1.09^{c} \pm 0.08$ & $0.77^{\mathrm{f}} \pm 0.09$ & $0.44^{\mathrm{g}} \pm 0.02$ \\
\hline
\end{tabular}

Means that are followed by different letters in a same line differ significantly according to Tukey's test (5\%).

The presence of the CLA isomers, 18:2 (c9,t11) and 18:2 (t10,c12) was observed in every sample. According to Rodrígues-Alcalá et al., ${ }^{26}$ these isomers were also detected in infant formulas. The c9,t11 isomer, also known as rumenic acid, is the predominant form of CLA found in naturally occurring foods. c9,t11 CLA comprises approximately $90 \%$ of the CLA found in ruminant meats and dairy products, and the $\mathrm{t} 10, \mathrm{c} 12$ isomer comprises the remaining $10 \%$. These values are equivalent to the obtained results of this study. Although several other isoforms of CLAs have been identified ( $\mathrm{t} 9, \mathrm{t} 11, \mathrm{c} 9, \mathrm{c} 11, \mathrm{t} 10, \mathrm{t} 11$ and $\mathrm{c} 10, \mathrm{c} 12)$, the $\mathrm{c} 9, \mathrm{t} 11$ and $\mathrm{t} 10, \mathrm{c} 12$ isomers appear to be the most biologically active. ${ }^{4,27}$ Researches indicate that these isomers possess different functions in the human body. For example c9,t11 is effective against cancer and t10,c12 possesses anti-carcinogenic, anti-obesity and antidiabetic properties. However, the biochemical mechanisms of CLA isomers are still questioned, with studies showing the possibility of metabolic changes and some health adverse effects. $^{28}$

Through the comparison of the described FA sums at the Table $3(\mathrm{P}<0.05)$, it is possible to verify that cow powdered milks from some brands showed equivalent CLA amounts in relation to goat milk, while in the rest of the brands greater CLA values were observed regarding the latter.
However, the CLA content in dairy products is affected by countless factors, such as those related to animals (aging, feeding and climate) and to food manipulation (processing, storage and raw material production). ${ }^{4,29}$

Regarding the short chain fatty acids (SCFA), represented by butyric (4:0), caproic (6:0), caprilic (8:0), capric (10:0) and caproleic (10:1n-1) acids, it was observed (Table 3) that the powdered milks from goats possess a significantly greater amount of these acids than the samples of bovine origin.

Another important result of this work was the TFA detection. It is known that the vaccenic acid is the main TFA present in the milk of ruminants and its synthesis naturally occurs through bio-hydrogenation reactions assisted by bacteria stored in the rumen. ${ }^{3}$ Many studies relate the TFA consumption with the risk of the occurrence of a number of diseases due to triacylglycerol accumulation in the sanguine plasma, leading to concerns about the ingestion of food with high TFA content. Because of that, many trans isomer identification techniques in food are under development, with the purpose of a better TFA regulation. ${ }^{30-33}$

According to the ANVISA RDC $359^{32}$ and $360^{33}$ resolutions (both from 2003), the commercialized powdered milks in Brazil may contain until $0.2 \mathrm{~g}$ of trans fat per $26 \mathrm{~g}$ portion so it can be labeled "trans fat free". ${ }^{32}$ 
Table 3. Fatty acid sums (mg per g of total lipids) and ratios of whole cow and goat powdered milks, Maringá (PR)

\begin{tabular}{|c|c|c|c|c|c|c|c|c|c|c|c|c|}
\hline Fatty acid & V1 & $\mathrm{V} 2$ & V3 & V4 & V5 & V6 & V7 & V8 & V9 & V10 & $\mathrm{C} 11$ & $\mathrm{C} 12$ \\
\hline$\overline{\text { SFA }}$ & $\begin{array}{c}631.13^{\mathrm{c}, \mathrm{d}} \\
\pm 0.78\end{array}$ & $\begin{array}{c}619.06^{\mathrm{c}, \mathrm{d}} \\
\pm 20.99\end{array}$ & $\begin{array}{l}593.10^{\mathrm{e}} \\
\pm 14.36\end{array}$ & $\begin{array}{c}623.39^{\mathrm{cd} d} \\
\pm 2.25\end{array}$ & $\begin{array}{c}638.23^{\mathrm{b}, \mathrm{c}} \\
\pm 3.18\end{array}$ & $\begin{array}{c}607.64^{\mathrm{d}, \mathrm{e}} \\
\pm 1.34\end{array}$ & $\begin{array}{r}635.05^{\circ} \\
\pm 3.86\end{array}$ & $\begin{array}{c}631.13^{c} \\
\pm 6.83\end{array}$ & $\begin{array}{c}657.38^{\mathrm{a}, \mathrm{b}} \\
\pm 4.05\end{array}$ & $\begin{array}{c}624.81^{\mathrm{c}, \mathrm{d}} \\
\pm 10.50\end{array}$ & $\begin{array}{c}664.93^{\mathrm{a}} \\
\pm 7.80\end{array}$ & $\begin{array}{c}657.19^{\mathrm{a}, \mathrm{b}} \\
\pm 5.01\end{array}$ \\
\hline MUFA & $\begin{array}{c}257.32^{\text {c,d,e }} \\
\pm 0.93\end{array}$ & $\begin{array}{c}258.26^{\mathrm{c}, \mathrm{d}, \mathrm{e}} \\
\pm 10.32\end{array}$ & $\begin{array}{c}300.03^{\mathrm{a}} \\
\pm 3.97\end{array}$ & $\begin{array}{c}262.38^{\mathrm{b}, c, d} \\
\pm 1.56\end{array}$ & $\begin{array}{c}252.89^{\mathrm{d}, \mathrm{e}} \\
\pm 2.67\end{array}$ & $\begin{array}{c}268.20^{\mathrm{b}, \mathrm{c}} \\
\pm 1.25\end{array}$ & $\begin{array}{c}267.01^{\mathrm{b}, \mathrm{c}} \\
\pm 1.80\end{array}$ & $\begin{array}{c}258.97^{\mathrm{cd}, \mathrm{e}} \\
\pm 5.16\end{array}$ & $\begin{array}{r}247.16^{\mathrm{e}} \\
\pm 3.65\end{array}$ & $\begin{array}{c}272.07^{\mathrm{b}} \\
\pm 8.14\end{array}$ & $\begin{array}{r}229.88^{\mathrm{f}} \\
\pm 7.19\end{array}$ & $\begin{array}{r}232.52^{\mathrm{f}} \\
\pm 5.24\end{array}$ \\
\hline PUFA & $\begin{array}{l}33.84^{c} \\
\pm 0.67\end{array}$ & $\begin{array}{l}46.67^{\mathrm{a}} \\
\pm 3.82\end{array}$ & $\begin{array}{l}32.37^{\mathrm{c}} \\
\pm 1.98\end{array}$ & $\begin{array}{l}42.22^{\mathrm{b}} \\
\pm 0.69\end{array}$ & $\begin{array}{l}26.88^{\mathrm{d}} \\
\pm 0.60\end{array}$ & $\begin{array}{l}42.16^{\mathrm{b}} \\
\pm 0.43\end{array}$ & $\begin{array}{l}33.44^{c} \\
\pm 0.69\end{array}$ & $\begin{array}{l}27.89^{d} \\
\pm 1.67\end{array}$ & $\begin{array}{l}25.20^{\mathrm{d}} \\
\pm 0.82\end{array}$ & $\begin{array}{l}32.87^{\circ} \\
\pm 1.38\end{array}$ & $\begin{array}{l}35.43^{c} \\
\pm 0.86\end{array}$ & $\begin{array}{l}40.02^{\mathrm{b}} \\
\pm 1.01\end{array}$ \\
\hline TFA & $\begin{array}{r}14.44^{\mathrm{fg} g} \\
\pm 0.90\end{array}$ & $\begin{array}{r}20.07^{\mathrm{b}, \mathrm{c}} \\
\pm 1.22\end{array}$ & $\begin{array}{c}21.54^{\mathrm{a}, \mathrm{b}} \\
\pm 0.92\end{array}$ & $\begin{array}{l}23.15^{\mathrm{a}} \\
\pm 1.23\end{array}$ & $\begin{array}{r}17.15^{\mathrm{d}, \mathrm{e}} \\
\pm 0.52\end{array}$ & $\begin{array}{l}23.20^{\mathrm{a}} \\
\pm 0.27\end{array}$ & $\begin{array}{c}18.86^{\mathrm{c}, \mathrm{d}} \\
\pm 0.43\end{array}$ & $\begin{array}{r}15.68^{\mathrm{e}, \mathrm{f}} \\
\pm 0.22\end{array}$ & $\begin{array}{c}14.36^{\mathrm{fgg}} \\
\pm 0.67\end{array}$ & $\begin{array}{l}19.50^{c} \\
\pm 1.30\end{array}$ & $\begin{array}{c}17.18^{\mathrm{d}, \mathrm{e}} \\
\pm 0.73\end{array}$ & $\begin{array}{l}13.00^{\mathrm{g}} \\
\pm 0.23\end{array}$ \\
\hline CLA & $\begin{array}{l}8.18^{\mathrm{e}, \mathrm{f}} \\
\pm 0.07\end{array}$ & $\begin{array}{l}9.20^{\mathrm{c}, \mathrm{d}} \\
\pm 0.30\end{array}$ & $\begin{array}{l}11.60^{\mathrm{a}} \\
\pm 0.29\end{array}$ & $\begin{array}{r}11.12^{\mathrm{a}, \mathrm{b}} \\
\pm 0.13\end{array}$ & $\begin{array}{l}9.06^{\mathrm{c}, \mathrm{d}} \\
\pm 0.21\end{array}$ & $\begin{array}{c}11.08^{\mathrm{a}, \mathrm{b}} \\
\pm 0.15\end{array}$ & $\begin{array}{l}9.47^{c} \\
\pm 0.15\end{array}$ & $\begin{array}{l}7.77^{\mathrm{f}} \\
\pm 0.15\end{array}$ & $\begin{array}{l}8.21^{\mathrm{e}, \mathrm{f}} \\
\pm 0.24\end{array}$ & $\begin{array}{l}10.85^{\mathrm{b}} \\
\pm 0.55\end{array}$ & $\begin{array}{l}8.60^{\mathrm{d}, \mathrm{e}} \\
\pm 0.40\end{array}$ & $\begin{array}{l}6.52^{\mathrm{g}} \\
\pm 0.22\end{array}$ \\
\hline$n-6$ & $\begin{array}{r}19.98^{\mathrm{b}, \mathrm{c}} \\
\pm 0.66\end{array}$ & $\begin{array}{l}31.45^{\mathrm{a}} \\
\pm 3.39\end{array}$ & $\begin{array}{c}15.82^{\mathrm{d}, \mathrm{e}, \mathrm{f}} \\
\pm 1.99\end{array}$ & $\begin{array}{l}21.78^{\mathrm{b}} \\
\pm 0.60\end{array}$ & $\begin{array}{r}13.71^{\mathrm{e}, \mathrm{f}} \\
\pm 0.58\end{array}$ & $\begin{array}{l}23.19^{b} \\
\pm 0.24\end{array}$ & $\begin{array}{c}17.69^{c, d} \\
\pm 0.62\end{array}$ & $\begin{array}{c}16.08^{\mathrm{d}, \mathrm{e}, \mathrm{f}} \\
\pm 1.62\end{array}$ & $\begin{array}{l}12.82^{\mathrm{f}} \\
\pm 0.43\end{array}$ & $\begin{array}{r}16.27^{\mathrm{d}, \mathrm{e}} \\
\pm 0.64\end{array}$ & $\begin{array}{l}22.38^{\mathrm{b}} \\
\pm 0.39\end{array}$ & $\begin{array}{l}29.29^{\mathrm{a}} \\
\pm 0.77\end{array}$ \\
\hline$n-3$ & $\begin{array}{l}4.64^{\mathrm{d}} \\
\pm 0.02\end{array}$ & $\begin{array}{l}5.17^{c} \\
\pm 0.28\end{array}$ & $\begin{array}{l}4.25^{\mathrm{e}} \\
\pm 0.14\end{array}$ & $\begin{array}{l}7.65^{\mathrm{a}} \\
\pm 0.07\end{array}$ & $\begin{array}{l}3.45^{\mathrm{f}} \\
\pm 0.04\end{array}$ & $\begin{array}{l}6.16^{\mathrm{b}} \\
\pm 0.19\end{array}$ & $\begin{array}{l}5.36^{c} \\
\pm 0.09\end{array}$ & $\begin{array}{l}2.70^{\mathrm{h}} \\
\pm 0.04\end{array}$ & $\begin{array}{l}3.05^{\mathrm{g}} \\
\pm 0.09\end{array}$ & $\begin{array}{l}4.65^{\mathrm{d}} \\
\pm 0.16\end{array}$ & $\begin{array}{l}1.77^{\mathrm{i}} \\
\pm 0.15\end{array}$ & $\begin{array}{l}2.59^{\mathrm{h}} \\
\pm 0.07\end{array}$ \\
\hline$n-6 / n-3$ & $\begin{array}{l}4.31^{\mathrm{d}} \\
\pm 0.16\end{array}$ & $\begin{array}{l}6.07^{\mathrm{c}} \\
\pm 0.33\end{array}$ & $\begin{array}{l}3.73^{\mathrm{d}, \mathrm{e}} \\
\pm 0.51\end{array}$ & $\begin{array}{l}2.85^{\mathrm{e}} \\
\pm 0.07\end{array}$ & $\begin{array}{l}3.97^{\mathrm{d}} \\
\pm 0.16\end{array}$ & $\begin{array}{l}3.76^{\mathrm{d}, \mathrm{e}} \\
\pm 0.13\end{array}$ & $\begin{array}{l}3.29^{\mathrm{d}, \mathrm{e}} \\
\pm 0.07\end{array}$ & $\begin{array}{l}5.95^{\mathrm{c}} \\
\pm 0.64\end{array}$ & $\begin{array}{l}4.19^{\mathrm{d}} \\
\pm 0.03\end{array}$ & $\begin{array}{l}3.49^{\mathrm{d}, \mathrm{e}} \\
\pm 0.05\end{array}$ & $\begin{array}{l}12.75^{\mathrm{a}} \\
\pm 1.10\end{array}$ & $\begin{array}{l}11.31^{\mathrm{b}} \\
\pm 0.21\end{array}$ \\
\hline PUFA/SFA & $\begin{array}{l}0.05^{\mathrm{cdd}} \\
\pm 0.00\end{array}$ & $\begin{array}{l}0.08^{\mathrm{a}} \\
\pm 0.00\end{array}$ & $\begin{array}{l}0.05^{\mathrm{c}, \mathrm{d}} \\
\pm 0.00\end{array}$ & $\begin{array}{l}0.07^{\mathrm{a}, \mathrm{b}} \\
\pm 0.00\end{array}$ & $\begin{array}{l}0.04^{\mathrm{e}} \\
\pm 0.00\end{array}$ & $\begin{array}{l}0.07^{\mathrm{a}} \\
\pm 0.00\end{array}$ & $\begin{array}{l}0.05^{\mathrm{d}} \\
\pm 0.00\end{array}$ & $\begin{array}{l}0.04^{\mathrm{e}} \\
\pm 0.00\end{array}$ & $\begin{array}{l}0.04^{\mathrm{e}} \\
\pm 0.00\end{array}$ & $\begin{array}{l}0.05^{\mathrm{d}} \\
\pm 0.00\end{array}$ & $\begin{array}{l}0.05^{\text {c.d }} \\
\pm 0.00\end{array}$ & $\begin{array}{l}0.06^{\text {b.c }} \\
\pm 0.00\end{array}$ \\
\hline SCFA & $\begin{array}{l}92.77^{\mathrm{c}} \\
\pm 1.43\end{array}$ & $\begin{array}{r}75.74^{\mathrm{d}, e} \\
\pm 7.48\end{array}$ & $\begin{array}{l}68.02^{\mathrm{e}} \\
\pm 3.74\end{array}$ & $\begin{array}{l}92.64^{c} \\
\pm 5.63\end{array}$ & $\begin{array}{c}80.77^{\mathrm{cd,de}} \\
\pm 1.24\end{array}$ & $\begin{array}{c}80.18^{\mathrm{c}, \mathrm{d}, \mathrm{e}} \\
\pm 2.46\end{array}$ & $\begin{array}{c}86.69^{\mathrm{c}, \mathrm{d}} \\
\pm 5.16\end{array}$ & $\begin{array}{r}73.51^{\mathrm{d}, \mathrm{e}} \\
\pm 1.31\end{array}$ & $\begin{array}{l}93.82^{\mathrm{c}} \\
\pm 1.38\end{array}$ & $\begin{array}{c}82.98^{\mathrm{c}, \mathrm{d}, \mathrm{e}} \\
\pm 6.95\end{array}$ & $\begin{array}{l}170.57^{\mathrm{a}} \\
\pm 14.33\end{array}$ & $\begin{array}{c}145.50^{\mathrm{b}} \\
\pm 7.82\end{array}$ \\
\hline MCFA & $\begin{array}{c}467.46^{\mathrm{b}, \mathrm{c}} \\
\pm 0.71\end{array}$ & $\begin{array}{l}472.04^{\mathrm{b}} \\
\pm 16.81\end{array}$ & $\begin{array}{r}460.95^{\mathrm{b}, \mathrm{c}} \\
\pm 11.76\end{array}$ & $\begin{array}{r}451.41^{\mathrm{c}} \\
\pm 2.20\end{array}$ & $\begin{array}{r}492.65^{\mathrm{a}} \\
\pm 4.53\end{array}$ & $\begin{array}{c}457.82^{\mathrm{b}, \mathrm{c}} \\
\pm 0.39\end{array}$ & $\begin{array}{c}463.68^{\mathrm{b}, \mathrm{c}} \\
\pm 4.88\end{array}$ & $\begin{array}{c}493.90^{\mathrm{a}} \\
\pm 6.66\end{array}$ & $\begin{array}{c}507.88^{\mathrm{a}} \\
\pm 6.68\end{array}$ & $\begin{array}{c}464.83^{\mathrm{b}, \mathrm{c}} \\
\pm 8.75\end{array}$ & $\begin{array}{r}404.72^{\mathrm{e}} \\
\pm 2.08\end{array}$ & $\begin{array}{r}427.94^{\mathrm{d}} \\
\pm 0.36\end{array}$ \\
\hline LCFA & $\begin{array}{c}357.77^{\mathrm{b}, \mathrm{c}} \\
\pm 1.46\end{array}$ & $\begin{array}{c}376.22^{\mathrm{a}, \mathrm{b}} \\
\pm 17.14\end{array}$ & $\begin{array}{r}396.53^{\mathrm{a}} \\
\pm 6.83\end{array}$ & $\begin{array}{r}383.94^{\mathrm{a}} \\
\pm 3.73\end{array}$ & $\begin{array}{c}344.59^{\mathrm{c}, \mathrm{d}} \\
\pm 5.36\end{array}$ & $\begin{array}{c}380.00^{\mathrm{a}} \\
\pm 2.58\end{array}$ & $\begin{array}{c}385.12^{\mathrm{a}} \\
\pm 2.73\end{array}$ & $\begin{array}{r}350.58^{c} \\
\pm 7.58\end{array}$ & $\begin{array}{c}328.03^{\mathrm{d}} \\
\pm 7.25\end{array}$ & $\begin{array}{l}381.93^{\mathrm{a}} \\
\pm 14.48\end{array}$ & $\begin{array}{r}354.95^{\mathrm{b}, \mathrm{c}} \\
\pm 12.54\end{array}$ & $\begin{array}{c}356.29^{\mathrm{b}, \mathrm{c}} \\
\pm 8.98\end{array}$ \\
\hline
\end{tabular}

Means that are followed by different letters in a same line differ significantly according to Tukey's test (5\%). SFA: saturated fatty acids, MUFA: monounsaturated fatty acids, PUFA: polyunsaturated fatty acids, TFA: trans fatty acids, CLA: conjugates linoleic acid, n-6 omega-6, n-3 omega-3, SCFA: short chain fatty acids, MCFA: medium chain fatty acids, LCFA: long chain fatty acids.

The analyses showed that all the samples possess very low values (Figure 1).

PUFAs regarded as beneficial to human health were also detected, especially linoleic (18:2n-6, LA) and alphalinolenic acids (18:3n-3, LNA), constituents of the $n-6$ and n-3 PUFA families, respectively (Figure 2). In accordance with the applied statistics $(\mathrm{P}<0.05)$, the LNA showed greater difference between the milk brands, but in the $\mathrm{n}-6$ series (FAs 18:2n-6 and 18:3n-6) this variation happened in a lesser degree.

Although studies involving the lipid quantification of milks obtained after thermal treatment are scarce in the literature, there are studies about the storage stability of powdered milks.

Romeu-Nadal et al..$^{34}$ compared the shelf life of long chain polyunsaturated fatty acids (LC-PUFAs) in supplemented and non- supplemented powdered milks, and it was noticed that their oxidative stability depends of how small is the PUFA content of them and of storage conditions, such as temperature control and storage time.

Rodriguez-Alcala et $a l .{ }^{26}$ relates that in the first storage year of powdered infant formulas, among the MUFAs

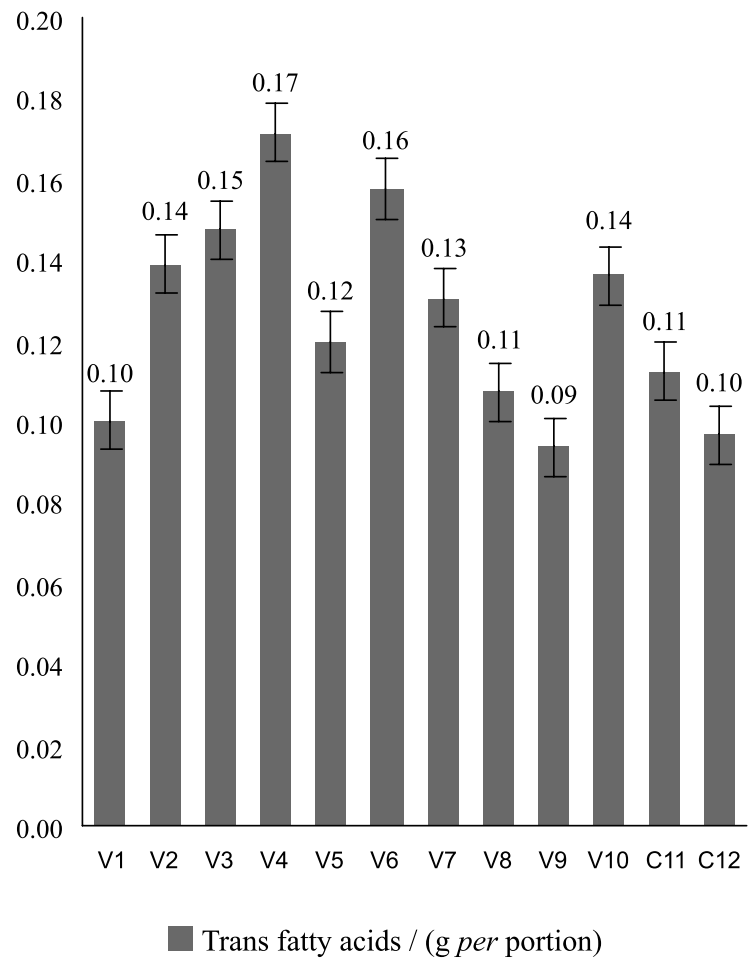

Figure 1. Amount of trans fatty acids (g per $26 \mathrm{~g}$ sample portion). 


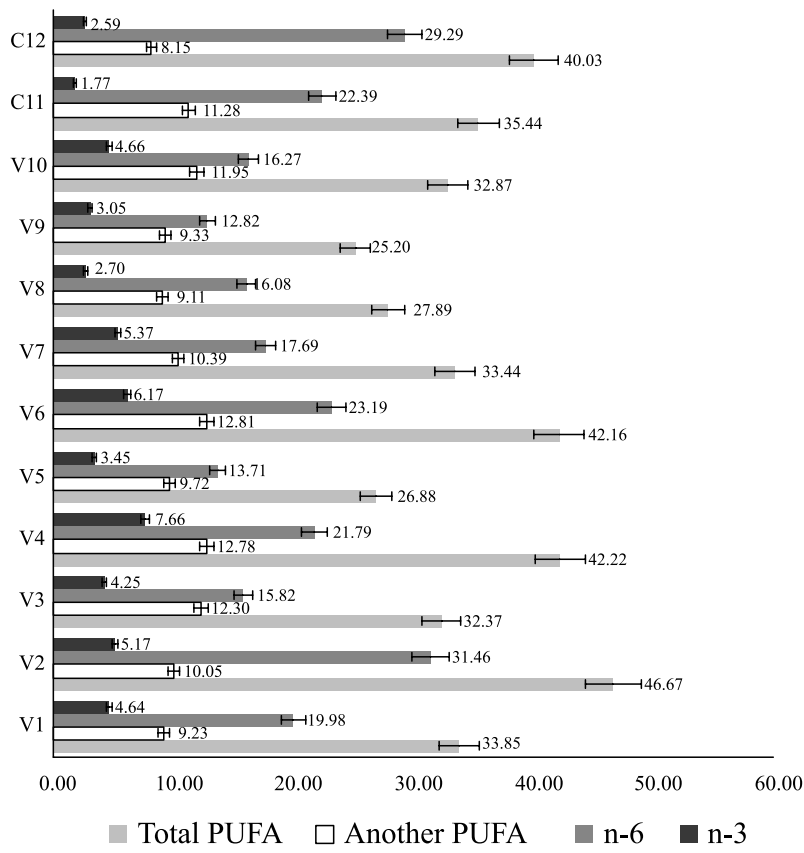

Figure 2. Comparison of polyunsaturated fatty acids (mg per $\mathrm{g}$ of total lipids), of whole cow and goat powdered milks.

and PUFAs, it was observed a gradual reduction of oleic and alpha-linolenic acid while the linoleic acid suffered significant decrease in the fourth year only. However, the long term storage only did small changes in the lipid constitution of the analyzed samples.

Researches proved that the PUFAs oxidation degree and its influence in the $n-6 / n-3$ ratio is related with the stability of lipids contained in foods. If the PUFAs are in a free state, their oxidation is more intense. ${ }^{12}$

Simopoulos ${ }^{35}$ relates that the optimal dose of $\mathrm{n}-6$ per $\mathrm{n}-3$, varies from $1 / 1$ to $4 / 1$. This conclusion is based on another medical research, which evaluated the relation of essential FAs ingestion with the healing rate of patients with chronic diseases.

Through this consideration, it was observed that between the ten brands of powdered milk from cow, eight are in accordance with the ratio stipulated by Simopoulos, ${ }^{35}$ whereas the ratios of goat powdered milks are significantly higher, leading to the statement that the consumption of goat powdered milk must be accompanied of other omega-3 sources, with the intention of decrease its $n-6 / n-3$ ratio.

However, much is discussed about the perspective of the $\mathrm{n}-6 / \mathrm{n}-3$ ratio, resulting in many recommendations which have been established by authors and health authorities, of different countries. WHO (World Health Organization) and FAO (Food and Agriculture Organization) suggest ratios from 5:1 to $10: 1 ;{ }^{36}$ Chardigny et al. from France mention 5:1, and Kris-Etherton et al. from Japan cite values between 2:1 and 4:1, according to Martin et $a l^{5}$
The establishment of this ratio exists because of the competition of n-6 and n-3 FA families for enzymes involved in FA chain desaturation and elongation reactions that start in the human body after FA ingestion. Although these enzymes possess greater affinity by $n-3 \mathrm{FAs}$, the conversion of LNA in LC-PUFAs is strongly affected by LA levels. ${ }^{37,38}$ These LC-PUFAs possess other important properties for the human health, and they are vitals for a range of metabolic functions that occurs in the human body. ${ }^{38}$

\section{Conclusions}

This study showed that even after the thermal treatment applied to powdered milks, the polyunsaturated fatty acids, especially the essentials linoleic and alpha-linolenic acids, are still present in the lipid fraction of the final product.

The $n-6 / n-3$ ratio was higher for the goat powdered milks. However they showed greater minerals and short chain fatty acid concentrations. There is not a great distinction of CLA contents between milk samples from cow and goat.

The trans fatty acid concentrations of all the analyzed milks were inferior to $0.2 \mathrm{~g}$ per portion, being in accordance with the actual Brazilian legislation. ${ }^{32,33}$

\section{Acknowledgments}

We gratefully acknowledge financial support from the National Council for Technological and Scientific Development (CNPq), process: 504106/2010-4.

\section{References}

1. Bates, C. J.; Prentice A.; Vitamins, Minerals and Essential Trace Elements. Drugs and Human Lactation, $1^{\text {st }}$ ed.; Elsevier Science Publishers B.V: Amsterdam, 1996.

2. Ceballos, L. S.; Morales, E. R.; Adarve, G. T.; Castro, J. D.; Matínez, L. P.; Sampelayo, M. R. S.; J. Food Compos. Anal. 2009, 22, 322.

3. Benjamin, S.; Spener, F.; Nutrition \& Metabolism 2009, 6:36.

4. Carvalho, E. B. T.; Melo, I. L. P.; Mancini-Filho, J.; Ciênc. Tecnol. Aliment. 2010, 30, 295.

5. Martin, C. A.; Almeida, V. V.; Ruiz, M. R.; Visentainer, J. E. L.; Matsushita, M.; Souza, N. E.; Visentainer, J. V.; Rev. Nutr. 2006, 19, 761.

6. Undurt, N. D.; Biotechnol. J. 2006, 1, 420.

7. Miles, E. A.; Banerjee, T.; Calder, P. C.; Prostaglandins, Leukotrienes Essent. Fatty Acids 2004, 70, 529.

8. Marques, A. C.; Valente, T. B.; Rosa, C. S.; Rev. Nutr. 2009, $22,283$. 
9. Davidek, J.; Velisek, J.; Pokorney, J.; Dev. Food Sci. 1990, 21, 448.

10. Ordóñez, J. A.; Tecnologia em Alimentos: Alimentos de Origem Animal, 1a. ed.; Artmed: Porto Alegre, 2005.

11. Kim, E. H-J.; Chen, X. G.; Pearce, D.; J. Food Eng. 2009, 94,163 .

12. 12. Araújo J. M. A.; Química de Alimentos: Teoria e Prática, 4a. ed.; UFV: Viçosa, MG, Brasil, 2008.

13. Waraho, T.; McClements, D. J.; Decker, E. A.; Trends Food Sci. Technol. 2011, 22, 3.

14. AOAC; Official Methods of Analysis, $15^{\text {th }}$ ed., Association of Official Analytical Chemistis: Arlington, 1990.

15. Folch, J.; Less, M.; Stanley, G. H. S.; J. Biol. Chem. 1957, 226 , 497.

16. Hartman, L.; Lago, R. C. A.; Laboratory Practices 1973, 22, 474.

17. Joseph, J. D.; Ackman, R. G.; J. AOAC Int. 1992, 75, 488.

18. Visentainer, J. V.; Quim. Nova 2012, 35, 274.

19. SAS Institute Inc.; SAS/STAT ${ }^{\circledast}$ 9.1.3.; Statistical Analysis System; SAS Institute: Cary, NC, USA, 2003.

20. Ministry of Agriculture, Livestock and Food Supplies (MAPA), Portaria $N^{\circ} 146,07 / 03 / 1996$. Avaiable at: http://extranet. agricultura.gov.br/sislegis-consulta/consultarLegislacao. do?operacao=visualizar\&id=1218 accessed in October 2012.

21. Trancoso, I. M.; Trancoso, M. A.; Martins, A. P. L; Roseiro, L. B.; Int. J. Dairy Technol. 2010, 63, 516.

22. Nijdam, J. J.; Langrish, T. A. G.; J. Food Eng. 2006, 77, 919.

23. Kim, E. H.-J.; Chen, X. G.; Pearce, D.; Colloids Surf., B 2005 , 46, 182.

24. Sieber, R.; Collomb, M.; Aeschlimann, A.; Jelen, P.; Eyer, H.; Int. Dairy J. 2004, 14, 1.

25. Bhattacharya, A.; Banu, J.; Rahman, M.; Causey, J.; Fernandes, G.; J. Nutr. Biochem. 2006, 17, 789.
26. Rodriguéz-Alcalá, L. M.; García-Martínez, M. C.; Cachón, F.; Marmesat, S.; Alonso, L.; Marquez-Ruiz, G.; Fontecha, J.; J. Agric. Food Chem. 2007, 55, 6533.

27. Kennedy, A.; Martinez, K.; Schmidt, S.; Mandrup, S.; LaPoint, K.; McIntosh, M.; J. Nutr. Biochem. 2010, 21, 171.

28. Nagao, K.; Yanagita, T.; J. Biosci. Bioeng. 2005, 100, 152.

29. Destaillats, F.; Golay, P. A.; Joffre, F.; de Wispelaere, M.; Hug, B.; Giuffrida, F. L.; Fauconnot, L.; Dionisi, F.; J. Chromatogr., A 2007, 1145, 222.

30. Dionisi, F.; Golay, P. A.; Fay, L. B.; Anal. Chim. Acta 2002, $465,395$.

31. Golay, P. A.; Dionisi, F.; Bernadette, H.; Giuffrida, F.; Destaillats, F.; Food Chem. 2006, 101, 1115.

32. http://www.mp.ba.gov.br/atuacao/ceacon/legislacao/alimentos/ resolucao_RDC_ANVISA_359_2003.pdf accessed in October 2012.

33. http://www.anvisa.gov.br/legis/resol/2003/rdc/360_03rdc.htm accessed in October 2012.

34. Romeu-Nadal, N.; Chávez-Servín, J. L.; Castellote, A. I.; Rivero, M.; López-Sabater, M. C.; Food Chem. 2007, 100, 756.

35. Simopoulos, A. P.; Biomed. Pharmacother. 2002, 56, 365.

36. World Health Organization (WHO) and Food and Agriculture Organization (FAO) http://www.fao.org/docrep/V4700E/ V4700E06.htm\#Essential fatty acids accessed in October 2012.

37. Russo, G. L.; Biochem. Pharmacol. 2009, 77, 937.

38. Barcelo-Coblijn, G.; Murphy, E. J.; Prog. Lipid Res. 2009, 48, 355 .

Submitted: July 5, 2012

Published online: October 26, 2012 